\title{
Circulating amino acids as fingerprints of visceral adipose tissue independent of insulin resistance: a targeted metabolomic research in women
}

\author{
Andrada A. Muresan ${ }^{1 *}$, Adriana Rusuํ, Raluca M. Pop ${ }^{2}$, Camelia L. Vonica ${ }^{1}$, \\ Nicolae Hancu ${ }^{1}$, Corina Bocsan ${ }^{2}$, Carmen Socaciu ${ }^{3}$, Cornelia G. Bala ${ }^{1}$, Gabriela \\ Roman $^{1}$
}

1. Diabetes and Nutrition, Iuliu Hatieganu University of Medicine and Pharmacy, Cluj-Napoca, Romania

2. Pharmacology, Toxicology and Clinical Pharmacology, Iuliu Hatieganu University of Medicine and Pharmacy, Cluj-Napoca, Romania

3. Chemistry, Biochemistry and Molecular Biology, University of Agricultural Sciences and Veterinary Medicine, Cluj-Napoca, Romania

\begin{abstract}
Introduction: Although obesity and its biomarkers have been intensively studied, little is known about the metabolomic signature of visceral adiposity independent of insulin resistance that frequently accompanies increased levels of visceral fat. Our study aimed to investigate specific changes in amino acid (AA) levels as biomarkers of increased visceral adiposity independent of insulin resistance, in healthy subjects. Methods: Forty-two adult women were included in this cross-sectional study. Serum samples were analyzed by AAs targeted metabolomics according to their visceral fat area $(<100 \mathrm{~cm} 2$ and $\geq 100 \mathrm{~cm} 2)$. Results: By corrected t-test and supervised partial least-squares discriminant analysis (PLS-DA) we identified 4 AAs that were significantly higher in the group with higher visceral fat: proline (variable importance in the projection [VIP] predicted value: 1.97), tyrosine (VIP: 2.21), cysteine (VIP: 1.19), isoleucine (VIP: 1.04; p-values <0.05). Also, glycine was significantly lower in the group with higher visceral fat (VIP: 1.65; p-value <0.05). All AAs identified were associated with visceral fat independent of homeostatic model assessment for insulin resistance (p-value for regression coefficients $<0.05$ ). Conclusion: Metabolic pathways that might be disrupted in persons with increased visceral fat are phenylalanine, tyrosine, and tryptophan biosynthesis; tyrosine metabolism; glycine, serine, and threonine metabolism; glyoxylate and dicarboxylate metabolism, and cysteine and methionine metabolism.
\end{abstract}

Keywords: metabolomics; amino acids; visceral fat; insulin resistance

Received: 10 $0^{\text {th }}$ September 2021; Accepted: $5^{\text {th }}$ October 2021; Published: $10^{\text {th }}$ October 2021

\footnotetext{
* Corresponding author: Andrada A. Muresan, Diabetes and Nutrition, Iuliu Hatieganu University of Medicine and Pharmacy, Cluj-Napoca, Romania. E-mail: andrada.oltean@gmail.com
} 


\section{Introduction}

Obesity has reached epidemic proportions; the World Health Organization estimated that in 2016 more than 650 million adults worldwide were living with obesity, accounting for $13 \%$ of the adult population [1]. Obesity is defined as an excessive or abnormal accumulation of adipose tissue within the whole body associated with an increased risk of chronic noncommunicable diseases [1]. A simple index of body fat commonly used in daily clinical practice to assess and classify obesity is body mass index (BMI), a weightfor-height ratio, according to which obesity is diagnosed at a value of $30 \mathrm{~kg} / \mathrm{m}^{2}$ [1].

Body fat distribution varies in persons with obesity, as well as in normal -weight persons, and studies have shown that, in terms of health-related risks, the regional distribution of adipose tissue is more important than total body fat or BMI [2]. Abdominal obesity or excessive visceral adiposity represents the intra-abdominal accumulation of adipose tissue into a greater amount as compared to limbs and hips and is associated with a systemic inflammatory state and insulin resistance, both linked to an increased risk of type 2 diabetes mellitus, cardiometabolic diseases, and premature death $[3,4]$. In the general population, the prevalence of excessive visceral adiposity is $>20 \%$ and is found not only in persons with obesity, but also in overweight or even normal weight persons [5]. Thus, a normal weight person with predominant abdominal fat accumulation will be considered as having abdominal obesity and increased morbidity and mortality risks, although the persons would not be considered obese according to their BMI [6]. The emergence of metabolomics has allowed the identification of biomarkers associated with obesity as well as biomarkers predictive of obesity-associated metabolic disorders and has provided useful information on the molecular pathways of this disease [7-9]. Because amino acids
(AAs) act as both metabolic modulators and substrates for the synthesis of proteins, they have been extensively studied in obesity both in observational and interventional studies [10]. Observational studies on the metabolomic changes associated with obesity and obesity-associated insulin resistance have found increased levels of AAs including branched-chained AAs (valine, leucine, and isoleucine) [11-13]. Furthermore, it has been shown that weight loss induced by either hypocaloric diets or bariatric surgery leads to lower levels of these AAs [14, 15].

Although a growing number of studies has tried to identify biomarkers associated with obesity, few have investigated AAs associated with visceral adiposity, and many have used histological samples of adipose tissue and evaluated the metabolome within adipose tissue, without concentrating on biomarkers easily accessible in clinical practice $[10,16]$. Given the fact that identified AAs associated with visceral adiposity were also linked to insulin resistance, in this study we aimed to investigate, in healthy subjects, specific changes in AA levels as the fingerprint of increased visceral adiposity independent of insulin resistance.

\section{Material and methods}

\section{Study design and participants}

This is a cross-sectional study performed between May 2017 and April 2021 in the Diabetes and Nutrition Department of the Iuliu Hatieganu University of Medicine and Pharmacy and Regina Maria Clinic Cluj-Napoca, Romania. We enrolled 42 consecutive clinically healthy adult women with a normal weight (BMI 18.5-24.9 $\mathrm{kg} / \mathrm{m}^{2}$ ) or obesity $\left(\mathrm{BMI} \geq 30 \mathrm{~kg} / \mathrm{m}^{2}\right)$, and who agreed to participate. We excluded women with a medical history of polycystic ovary syndrome, thyroid dysfunction, menopause, diabetes, dyslipidemia, hypertension, pregnancy, and/or lactation. 
The study protocol, information sheet, and informed consent form were approved by the Ethics Committee of the Iuliu Hatieganu University of Medicine and Pharmacy of Cluj-Napoca, Romania (No 318/ 21.07.2016). The study was conducted according to the International Conference on Harmonization Good Clinical Practice Guidelines and the Declaration of Helsinki. Written informed consent was obtained from all participants.

\section{Study assessments and data collection}

All study assessments were performed in the morning, in fasting conditions. Medical history and drug and food supplement use were assessed via interviews. Anthropometric indices (height, weight, waist circumference, and hip circumference) were measured with patients wearing light clothes. The BMI was calculated as weight $(\mathrm{kg}) /$ square of height $(\mathrm{m})$. The body composition analysis was performed by bioelectric impedance using an InBody 720 device (Biospace Co., South Korea). Parameters evaluated by bioelectric impedance analysis were VFA, body fat mass, skeletal muscle mass, and percentage of body fat. Blood pressure was measured after 5 min of rest, in a sitting position.

Blood samples were collected after at least $8 \mathrm{~h}$ of fasting and were used for the assessment of biochemical parameters (plasma glucose, total cholesterol, HDL-cholesterol, serum triglycerides, creatinine, alanine aminotransferase, aspartate aminotransferase), insulinemia, and targeted metabolomic analysis (serum AA levels). Blood samples were collected using serum clot activator collection tubes. Samples for the biochemical parameters were analyzed on the day of collection using commercially available enzymatic methods. Blood samples for insulinemia and metabolomic analysis were kept at room temperature for $30 \mathrm{~min}$ to allow clotting and centrifuged at $2000 \mathrm{rpm}$ for 10 minutes to separate serum which was frozen until assessment. An es- timated glomerular filtration rate was calculated using MDRD Study Equation formula, available online at: http://www.mdrd.com (last accessed 23 July 2021).

Insulinemia was measured by a commercially available ELISA sandwich test, according to the manufacturers' instructions (DIAsource ImmunoAssays S.A., Louvain-la-Neuve, Belgium). Insulin resistance was assessed using homeostatic model assessment for insulin resistance (HOMA-IR) calculated as fasting insulin $\mathrm{x}$ fasting glycemia/405 [17].

\section{Serum AA levels measurement}

The targeted metabolomic approach used for AA quantification in all serum samples was previously described [18]. Thermo ScientificTM UHPLC UltiMate 3000 equipped with a quaternary pump Dionex delivery system (Thermo Fisher Scientific, USA) and mass spectrometry (MS) detection with the MaXis Impact 2012 version (Bruker Daltonics, MA, USA) was used to quantify targeted AAs by ultrahigh performance liquid chromatography (UHPLC) coupled with electrospray ionization-quadrupole-time of MS in positive mode (UHPLC-QTOF -[ESI+]-MS). Before the analysis, the system was calibrated using a mixture kit of 20 pure AAs to assess the linearity and sensitivity of the method. The analysis and data processing were performed in the Research and Development Center for Applied Biotechnology in Diagnosis and Molecular Therapies, Cluj-Napoca, Romania.

All serum samples were thawed at room temperature and homogenized before analysis. According to the protocol, $0.4 \mathrm{ml}$ of trichloroacetic acid were added to a volume of $0.4 \mathrm{ml}$ serum for deproteinization. After 30 minutes of storage at $4^{\circ} \mathrm{C}$, the samples were centrifuged at $12,500 \mathrm{rpm}$ for $5 \mathrm{~min}$. The supernatant was filtered through $0.2 \mu \mathrm{m}$ Nylon filters. The filtrate was used for UHPLC-Q-TOF -[ESI+]-MS analysis.

The solvents used for UHPLC analysis were 
acetonitrile (Merck, Germany), sodium and ammonium formate (99\% purity) (Alpha Aesar, Germany), and $\mathrm{HClO}_{4}$ (Merck, Germany). Chromatographic separation was performed on $50 \times 3$ mm Intrada Amino Acid commercially available columns (Imtakt, Kyoto, Japan). For separation, we used a gradient of mobile phases of acetonitrile/formic acid (100/0.3, v/v) (A), and acetonitrile/ammonium formate $100 \mathrm{mM}(20 / 80$, $\mathrm{v} / \mathrm{v})(\mathrm{B})$. The gradient program was: B0\% (0-2.5 $\mathrm{min}) ; \mathrm{B} 17 \%$ at $6.5 \mathrm{~min}, \mathrm{~B} 100 \%$ at $12 \mathrm{~min}$. The flow was $0.6 \mathrm{ml} / \mathrm{min}$, and the sample volume 5 microliters.

The mass spectrometer with set parameters was used for the detection of AAs: ESI + fragmentation, nebulizer pressure of 2.8 bar, nitrogen flow $12 \mathrm{~L} / \mathrm{min}$, and temperature of $300^{\circ} \mathrm{C}$. Calibration of the spectrometer was done with sodium formate solution injected in the system before each run.

Nineteen AAs of interest were identified, quantified, and divided into essential (histidine, isoleucine, leucine, valine, lysine, methionine, phenylalanine, threonine, tryptophan), non-essential, and conditional essential (asparagine, glutamine, glycine, proline, serine, tyrosine, alanine, aspartic acid, cysteine, glutamic acid).

To quantify AA concentrations, three-point calibration curves were performed for each AA assessed in the first step, using the AA kit according to the technical specifications of the method applied by the producer of Intrada columns. Homoarginine, methionine-D3, and homophenylalanine were used as internal standards. For each AA, the curve factor $F=$ Signal area of the UHPLC chromatogram (A)/ $50 \mu \mathrm{M}$ concentration was determined. This factor $\mathrm{F}$ was used to calculate the concentration $\mathrm{C}$ of each AA in the sera using the QUANT ANALYSIS 2.2 system (Bruker Daltonics, Germany), which considered the areas of each AA (A) in the chromatogram and the curve factor (F), using the formula $C$ $(\mu \mathrm{M})=\mathrm{A} \times \mathrm{F}$. All measurements were performed in duplicate, and the average concentrations were reported.

Instrument control (UHPLC, MS) was done with specific Bruker software: OTofControl 3.2, HyStar 3.2, and data were processed with Data Analysis 4.2 and Quantitative Analysis 2.2 software (Bruker Daltonics, MA, USA).

Reference ranges used for AAs can be accessed at the following link: https://www.synevo.ro/ shop/aminoacizi-in-plasma/.

\section{Statistical analysis}

Participants were divided into 2 groups based on their visceral fat area (VFA): $\geq 100 \mathrm{~cm}^{2}$ and $<100 \mathrm{~cm}^{2}$. Statistical analysis of metabolomic data was performed using Metaboanalyst 5.0 software dedicated to metabolomics analysis. First, AA levels were normalized by log transformation. For the comparison of the metabolites between the 2 groups, a t-test with correction for false discovery rate (FDR) was performed. AAs with an FDR corrected p-value $<0.05$ were considered statistically different between groups. Intrinsic variation of AAs studied and screening of the potential biomarkers for increased VFA was performed by unsupervised principal component analysis (PCA) and supervised partial leastsquares discriminant analysis (PLS-DA). These procedures allow data summarization in fewer variables (scores) which are a weighted average of the original variables. For both methods, AAs with a |factor loading $\mid \geq 0.3$ were considered to contribute significantly to it. PLS-DA based on multivariate regression techniques allowed us to model the variations of metabolites in the 2 groups. The PLS-DA models obtained were validated by permutation and cross-validation (10fold CV), and the goodness of fit was assessed by calculation of $\mathrm{R}^{2}$ (evaluating the explanation of the data variations) and $\mathrm{Q}^{2}$ (an estimate of the predictive ability of the models). The best-fitted model was used to compute the VIP scores for all AAs; metabolites with VIP values $>1$ and an 
FDR p-value in t-test of $<0.05$ were selected for the pathway analysis. Also, to improve data visualization, a heatmap was generated based on hierarchical cluster analysis, by using the $\mathrm{Eu}-$ clidean for distance measures and Ward's linkage for the clustering algorithm.

To understand the impact of VFA on human metabolism, pathway analysis was also performed in Metaboanalyst 5.0 which combines results from pathway enrichment analysis with pathway topology analysis. By enrichment analysis, an FDR corrected p-value was computed for each pathway, and pathway topology analysis allowed calculation of the pathway impact score (an estimate of the importance of a given pathway relative to a global metabolic network). To filter important pathways, a cut-off value of 0.1 for pathway impact score was used [19]. Human metabolic pathways in the Kyoto Encyclopedia of Genes and Genomes (KEGG) database were used as reference.

Statistical analysis of anthropometric and biochemical parameters was performed in SPSS-PC 20.0 (SPSS Inc., Chicago, IL, USA). Data were presented as means and standard deviation for continuous variables with normal distribution, as median (quartiles 1 and 3) for continuous variables with non-normal distribution, and as number (percentage) for categorical variables. To compare the variables between the VFA and anthropometric indices, we used the student t-test and the median test for continuous variables, and the chi-square test for frequencies. The association between VFA and identified AAs was assessed using linear regression analysis. A p-value $<0.05$ was considered statistically significant.

\section{Results}

Demographic and clinical data of participants We included 42 women (24 with VFA $>100 \mathrm{~cm}^{2}$ and 18 with VFA $<100 \mathrm{~cm}^{2}$ ). The group with VFA $\geq 100 \mathrm{~cm}^{2}$ had significantly higher BMI, waist circumference, homeostatic model assessment for insulin resistance, LDL cholesterol, body fat mass, skeletal muscle mass, and percentage of body fat ( $p<0.05$ for these variables). No difference between the study groups was observed for age, fasting plasma glucose, triglycerides, creatinine, and liver enzymes (Table 1). None of the participants were using food supplements (including those containing AAs).

Identification of AAs according to VFA groups Comparison of metabolomic data between groups with VFA $\geq 100 \mathrm{~cm}^{2}$ and $<100 \mathrm{~cm}^{2}$ showed that 7 essential AAs and 6 non-essential AAs were significantly different. The group with VFA $\geq 100 \mathrm{~cm}^{2}$ showed higher levels of essential (valine, isoleucine, methionine, phenylalanine, tryptophan, lysine, histidine), conditional, and non-essential AAs (proline, tyrosine, alanine, cysteine, serine), and significantly lower levels of glycine (non-essential AA) when compared to women in the VFA $<100 \mathrm{~cm}^{2}$ group (Table 2).

By unsupervised PCA, which allowed exploration of the general trend of AA concentrations, we identified 5 factors explaining $76.6 \%$ of the variance between groups, showing good clustering as indicated in the three-dimensional score plot (Figure 1A). Metabolites with the highest loading values on Factor 1 were cysteine, proline, tyrosine, and glycine; those with the highest loading on Factor 2 were proline, lysine, glutamic acid, and glycine; on Factor 3 cysteine, lysine, valine, glutamine, and glutamic acid; on Factor 4 methionine, histidine, aspartic acid, cysteine, glycine, and proline; on Factor 5 histidine, methionine, phenylalanine, glutamine, and asparagine.

The identification of AAs discriminating between the groups with VFA $<100 \mathrm{~cm}^{2}$ and $\geq 100$ $\mathrm{cm}^{2}$ was performed by PLS-DA. The best-fitting PLSA-DA model had 5 major components, explaining $70.9 \%$ of the variance in AA levels, with a principal component 1 (PC1) of $46.5 \%, \mathrm{PC} 2$ of $11.5 \%$, PC3 of $6 \%$, PC4 of $4.2 \%$, and PC5 
Table 1. Participants' characteristics according to visceral fat area category.

\begin{tabular}{|c|c|c|c|}
\hline & $\begin{array}{c}\mathrm{VFA} \geq 100 \mathrm{~cm}^{2} \\
\mathrm{~N}=18\end{array}$ & $\begin{array}{c}\mathrm{VFA}<100 \mathrm{~cm}^{2} \\
\mathrm{~N}=24\end{array}$ & p-Value \\
\hline Age, years & $36.5 \pm 8.0$ & $33.8 \pm 6.1$ & 0.236 \\
\hline BMI, $\mathrm{kg} / \mathrm{m}^{2}$ & $36.4 \pm 7.6$ & $21.3 \pm 1.3$ & $<0.001$ \\
\hline Waist, cm & $105.1 \pm 13.6$ & $76.8 \pm 5.8$ & $<0.001$ \\
\hline $\mathrm{FPG}, \mathrm{mg} / \mathrm{dL}$ & $91.4 \pm 8.2$ & $90.4 \pm 8.5$ & 0.703 \\
\hline HOMA-IR & $3.2(2.6 ; 3.6)$ & $2.6(2.3 ; 2.7)$ & 0.010 \\
\hline LDL cholesterol, $\mathrm{mg} / \mathrm{dL}$ & $124.9 \pm 31.3$ & $98.4 \pm 20.9$ & 0.004 \\
\hline HDL cholesterol, mg/dL & $52.4 \pm 12.0$ & $62.8 \pm 10.0$ & 0.006 \\
\hline Triglycerides, mg/dL & $97.5 \pm 45.8$ & $81.2 \pm 40.5$ & 0.246 \\
\hline Creatinine, $\mathrm{mg} / \mathrm{dL}$ & $0.7 \pm 0.1$ & $0.7 \pm 0.1$ & 0.393 \\
\hline eGFR, $\mathrm{mL} / \mathrm{min} / 1.73 \mathrm{~m}^{2}$ & $112.6 \pm 11.6$ & $113.8 \pm 11.8$ & 0.800 \\
\hline ASAT U/L & $15.5 \pm 2.4$ & $17.1 \pm 2.9$ & 0.063 \\
\hline ALAT U/L & $13.4 \pm 4.7$ & $14.8 \pm 5.0$ & 0.372 \\
\hline SBP, $\mathrm{mmHg}$ & $113.6 \pm 11.5$ & $111.7 \pm 9.6$ & 0.632 \\
\hline $\mathrm{DPB}, \mathrm{mmHg}$ & $79.2 \pm 6.5$ & $74.3 \pm 7.0$ & 0.075 \\
\hline Body fat mass, $\mathrm{kg}$ & $44.8 \pm 14.1$ & $15.7 \pm 3.2$ & $<0.001$ \\
\hline SMM, kg & $29.4 \pm 4.6$ & $23.3 \pm 2.6$ & $<0.001$ \\
\hline PBF, \% & $45.3 \pm 5.7$ & $26.8 \pm 4.0$ & $<0.001$ \\
\hline
\end{tabular}

$\mathrm{N} / \mathrm{n}$ (\%), number (percentage) of participants; Q, quartile; BMI, body mass index; FPG, fasting blood glucose; ASAT, aspartate aminotransferase; ALAT, Alanine aminotransferase; eGFR, estimated glomerular filtration rate; HOMA-IR, homeostatic model assessment for insulin resistance; SBP, systolic blood pressure; DBP, diastolic blood pressure; SMM, skeletal muscle mass; PBF, percent body fat.

of $2.7 \%$. AAs with the highest loading on PC1 were cysteine, proline, tyrosine, and glycine; on PC2 proline, isoleucine, lysine, glutamic acid, and glycine; on PC3 lysine, glutamine, glutamic acid, and serine; on PC4 glutamic acid, serine, glutamine, and lysine; on PC5 valine, glutamine, proline, and glutamic acid. Three-dimensional PLS-DA scores plot of serum samples (Figure 1B) showed a complete separation between the groups VFA $<100 \mathrm{~cm}^{2}$ and $\geq 100 \mathrm{~cm}^{2}$ suggesting

Table 2. AA concentrations ( $\mu \mathrm{mol} / \mathrm{l})$, in different groups, as identified by t-tests and VIP scores.

\begin{tabular}{|c|c|c|c|c|}
\hline & $\begin{array}{c}\text { VFA } \geq 100 \mathrm{~cm}^{2} \\
N=18\end{array}$ & $\begin{array}{c}\begin{array}{c}\text { VFA }<100 \mathrm{~cm}^{2} \\
N=24\end{array}\end{array}$ & $\begin{array}{c}\text { FDR Corrected } \\
\text { p-Value }\end{array}$ & VIP scores \\
\hline Proline & $292.5(245.5 ; 346.8)$ & $140.3(127.1 ; 184.5)$ & $<0.001$ & 1.98 \\
\hline Tyrosine & $91.2(73.5 ; 97.1)$ & $37.8(35 ; 49.2)$ & $<0.001$ & 2.21 \\
\hline Glycine & $138.3(108 ; 186.4)$ & $254.4(215.4 ; 276.5)$ & $<0.001$ & 1.65 \\
\hline Cysteine & $45.3(38.9 ; 52.6)$ & $32.0(24.6 ; 35.5)$ & 0.00024619 & 1.19 \\
\hline Isoleucine & $97.2(90.8 ; 107.5)$ & $67.3(53.8 ; 82)$ & 0.00011604 & 1.04 \\
\hline Valine & $238.3(221.4 ; 272.7)$ & $178.8(162.6 ; 194.4)$ & $<0.001$ & 0.94 \\
\hline Methionine & $34(27.6 ; 37.6)$ & $25.2(18.3 ; 29.1)$ & 0.00071634 & 0.99 \\
\hline Alanine & $394.8(378.1 ; 418.5)$ & $312.8(288.8 ; 320.7)$ & $<0.001$ & 0.78 \\
\hline Phenylalanine & $111.9(100.6 ; 122.5)$ & $87(70.8 ; 97.6)$ & 0.0014978 & 0.8 \\
\hline Lysine & $249.9(212.5 ; 286.5)$ & $189(146.2 ; 263.7)$ & 0.015795 & 0.79 \\
\hline Serine & $92.4(87 ; 101.4)$ & $76.1(68.5 ; 874)$ & 0.00028937 & 0.61 \\
\hline Histidine & $77.6(65.9 ; 84.8)$ & $56.2(52 ; 65.3)$ & 0.016793 & 0.57 \\
\hline Tryptophan & $80.9(75.3 ; 86.9)$ & $72(61.3 ; 76.9)$ & 0.002266 & 0.42 \\
\hline
\end{tabular}

$\mathrm{N}$, number of participants; FDR, false discovery rates; VIP, variable importance in the projection. 
a good description and prediction of this model. The results displayed by score plots were confirmed by cross-validation and permutation tests. In cross-validation the accuracy of this model was $0.9, \mathrm{R}^{2}$ and $\mathrm{Q}^{2}$ were 0.88 and 0.55 , respectively. By the permutation test, we obtained an empirical $p$-value $<0.01$ for prediction accuracy during training and separation distance (100 permutations performed). By PLS-DA loadings, 5 metabolites with VIP scores $>1$ and thus with significant variation between groups were identified and retained for pathway analysis: tyrosine (VIP predicted value: 2.21), proline (VIP predicted value: 1.97 ), glycine (VIP predicted value: 1.65), cysteine (VIP predicted value: 1.19), and isoleucine (VIP predicted value: 1.04) (Table 2).

The results of the hierarchical clustering were further visually represented in a heatmap for individual and group samples (Figure 2).

\section{Metabolic pathway analysis}

For the pathway analysis, we mapped correlated metabolic pathways with the 5 metabolites with FDR corrected $p$-value $<0.05$, and VIP $>1$ listed in Table 1. We identified 17 pathways sig-

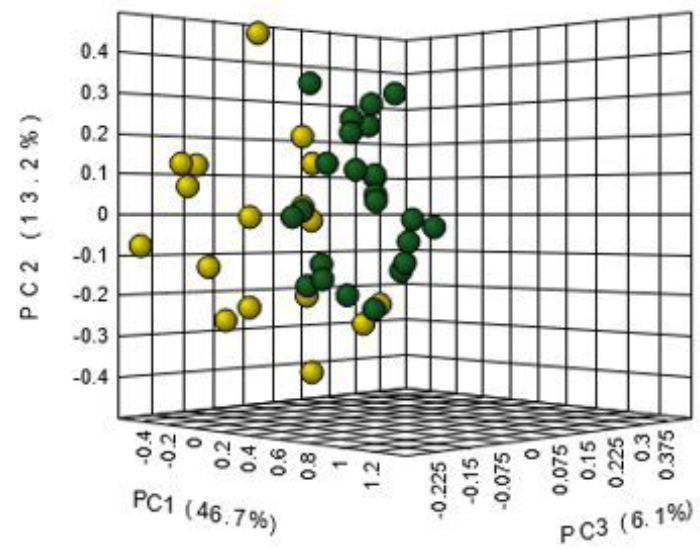

(a) nificantly enriched when comparing VFA $<100$ $\mathrm{cm}^{2}$ and VFA $\geq 100 \mathrm{~cm}^{2}$ (with an FDR corrected p-value $<0.05$ ). Of these, 5 metabolic pathways had impact scores $\geq 0.1$ : phenylalanine, tyrosine, and tryptophan biosynthesis; tyrosine metabolism; glycine, serine, and threonine metabolism; glyoxylate and dicarboxylate metabolism, and cysteine and methionine metabolism (Table 3).

Association of the visceral fat area with identified amino acids - influence of insulin resistance As HOMA-IR had a statistically significant difference between groups and previous research showed the association of this variable with the level of identified AAs [13, 18] we performed a linear regression adjusted for HOMA-IR with the 5 AAs with VIP score $>1$ by PLSDA as dependent variables and VFA as an independent variable. All AAs included in this analysis were associated with VFA independent of HOMA-IR (p-value for all regression coefficients $<0.001$ ) and most of the associations were positive, except for glycine which was inversely associated with VFA (Table 4).

Although BMI, percentage of body fat, and body fat mass also had statistically significant differences between groups, variance inflation factors

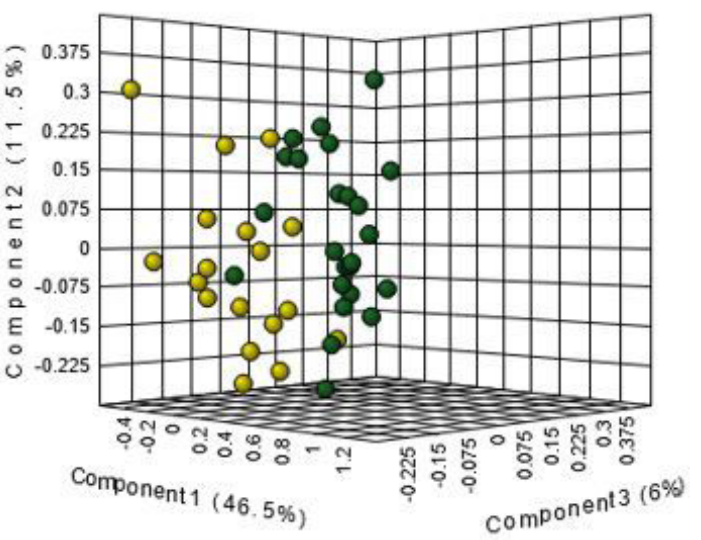

(b)

Fig. 1. Principal component analysis (a) and supervised partial least-squares discriminant analysis (b) score plots between the selected principal components. VFA $<100 \mathrm{~cm}^{2}$ group is depicted as yellow circles; $V F A \geq 100 \mathrm{~cm}^{2}$ group is depicted as dark green circles. 


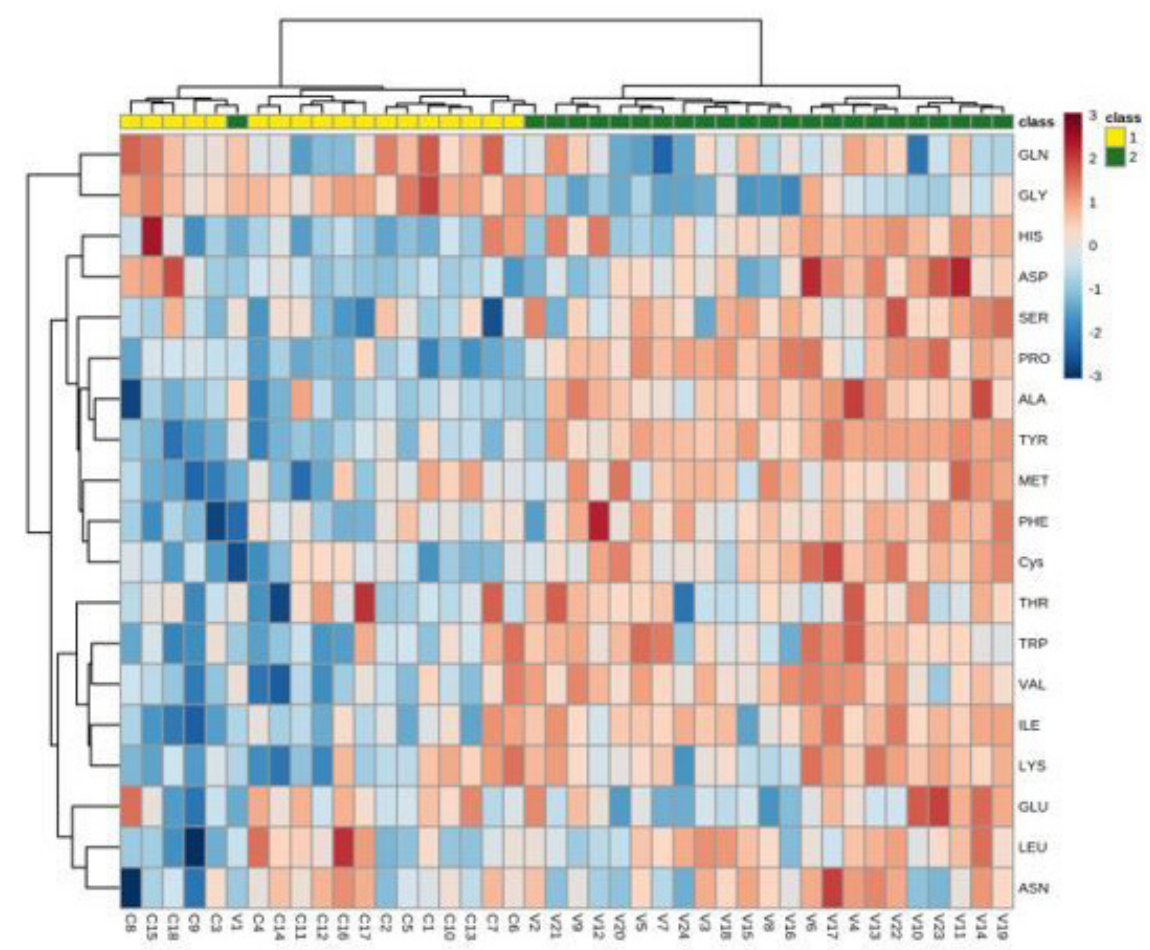

Fig. 2. Heatmap of targeted metabolomic analysis for the correlation between analyzed amino acids and individual samples. Rows, amino acids; columns, individual samples. Color key shows the metabolite expression value: lowest (blue) and highest (red); 1 refers to $\mathrm{VFA}<100 \mathrm{~cm}^{2}$ group; 2 refers to VFA $\geq 100$ $\mathrm{cm}^{2}$ group.

Table 3. Pathway analysis of serum metabolomics data.

\begin{tabular}{lcccc}
\hline Pathway name & Hits & $\begin{array}{c}\text { Raw } \\
\text { p-value }\end{array}$ & $\begin{array}{c}\text { FDR-corrected } \\
\text { p-value }\end{array}$ & Impact \\
\hline Aminoacyl-tRNA biosynthesis & 5 & $<0.001$ & $<0.001$ & 0.00 \\
\hline Arginine and proline metabolism & 1 & $<0.001$ & $<0.001$ & 0.08 \\
\hline Phenylalanine, tyrosine and tryptophan biosynthesis & 1 & $<0.001$ & $<0.001$ & 0.50 \\
\hline Tyrosine metabolism & 1 & $<0.001$ & $<0.001$ & 0.14 \\
\hline Ubiquinone and other terpenoid-quinone biosynthesis & 1 & $<0.001$ & $<0.001$ & 0.00 \\
\hline Phenylalanine metabolism & 1 & $<0.001$ & $<0.001$ & 0.00 \\
\hline Glycine, serine, and threonine metabolism & 2 & $<0.001$ & $<0.001$ & 0.25 \\
\hline Glutathione metabolism & 2 & $<0.001$ & $<0.001$ & 0.09 \\
\hline Glyoxylate and dicarboxylate metabolism & 1 & $<0.001$ & $<0.001$ & 0.11 \\
\hline Primary bile acid biosynthesis & 1 & $<0.001$ & $<0.001$ & 0.01 \\
\hline Porphyrin and chlorophyll metabolism & 1 & $<0.001$ & $<0.001$ & 0.00 \\
\hline Valine, leucine, and isoleucine degradation & 1 & $<0.001$ & $<0.001$ & 0.00 \\
\hline Valine, leucine, and isoleucine biosynthesis & 1 & $<0.001$ & $<0.001$ & 0.00 \\
\hline Cysteine and methionine metabolism & 1 & $<0.001$ & $<0.001$ & 0.10 \\
\hline Taurine and hypotaurine metabolism & 1 & $<0.001$ & $<0.001$ & 0.00 \\
\hline Thiamine metabolism & 1 & $<0.001$ & $<0.001$ & 0.00 \\
\hline Pantothenate and CoA biosynthesis & 1 & $<0.001$ & $<0.001$ & 0.00 \\
\hline
\end{tabular}


Table 4. Standardized $\beta$-estimates from linear regression analyses with the visceral fat area as exposure and identified amino acids as the outcome.

\begin{tabular}{lcc}
\hline Amino acids & $\begin{array}{c}\text { Standardized } \\
\boldsymbol{\beta} \text {-estimates }\end{array}$ & p-value \\
\hline Proline & 0.773 & $<0.001$ \\
\hline Tyrosine & 0.804 & $<0.001$ \\
\hline Glycine & -0.696 & $<0.001$ \\
\hline Cysteine & 0.624 & $<0.001$ \\
\hline Isoleucine & 0.621 & $<0.001$ \\
\hline
\end{tabular}

in the models including these variables and VFA were $>10$ suggesting collinearity. Thus, these variables were not included in the regression models as covariables.

\section{Discussion}

In this research, we aimed to assess the biomarker signature of increased visceral fat using a targeted metabolomic approach. We identified 4 AAs that were significantly higher (proline, tyrosine, cysteine, isoleucine) and 1 AA (glycine) that was significantly lower in the group with higher visceral fat. Furthermore, we showed that these AAs remained associated with visceral fat independent of insulin resistance. Corresponding metabolic pathways that might be disrupted in persons with increased visceral adipose tissue were phenylalanine, tyrosine, and tryptophan biosynthesis; tyrosine metabolism; glycine, serine, and threonine metabolism; glyoxylate and dicarboxylate metabolism, and cysteine and methionine metabolism.

While many studies have assessed specific plasma metabolite alterations in obesity, few have focused on biomarkers specific for visceral adipose tissue and have aimed to identify AAs associated with visceral fat [10, 16, 20-23]. In a sample of 59 women (lean, overweight, and obese), Boulet et al. [21] showed that total AAs and branchedchain AAs were associated with visceral adipose tissue. In 2 other studies enrolling only women with overweight and obesity, of the AAs assessed by targeted metabolomics, glutamine, leucine/ isoleucine, phenylalanine, and tyrosine or alanine, glycine, glutamate, tryptophan, tyrosine, and branched-chain AAs were associated with visceral fat $[22,23]$. These results support our findings regarding the identification of proline, tyrosine, cysteine, isoleucine, and glycine. The difference in the AAs identified in our study as compared to the previous ones may be explained by the structure of the sample enrolled - normal weight and obese persons in our study and only persons with obesity in the previous studies. Branched-chain AAs (leucine, isoleucine, and valine) have been linked to obesity and visceral adipose tissue, insulin resistance, and increased risk of type 2 diabetes, dyslipidemia, and cardiovascular diseases [12, 13, 24-27]. In our study, we also found an increased level of isoleucine in participants with higher VFA, free of type 2 diabetes. Given that increased levels of branched-chain AAs have been observed in multiple chronic non-communicable diseases linked to metabolism, it has been suggested that changes in metabolic profiling that involves these AAs are not specific for a certain disease, but rather characterize a metabolic change associated with visceral adiposity $[7,28]$.

We also found increased levels of tyrosine in our group with increased VFA, and the levels of this AA were associated with VFA independent of insulin resistance. Tyrosine is a non-essential AA produced endogenously by hydroxylation of phenylalanine and metabolized by transamination of tyrosine to p-hydroxyphenylpyruvate, a reaction catalyzed by tyrosine aminotransferase in the liver [29]. A known inhibitor of tyrosine aminotransferase is cysteine and, thus, increased cysteine levels are associated with increased tyrosine levels [29]. Two previous studies performed in obese Japanese persons and healthy Caucasian women also reported higher tyrosine levels in participants with increased visceral ad- 
ipose tissue, thus supporting our findings [22, 23]. In metabolomic studies, tyrosine was typically associated with insulin resistance and a strong predictor of type 2 diabetes [30]. It has been hypothesized that in the insulin resistance states, increased levels of tyrosine and cysteine precede alterations of branched-chained AAs [29]. However, in our research, the association of tyrosine and cysteine levels with VFA was independent of insulin resistance, thus supporting a role of visceral adiposity in determining the levels of these AAs.

Previous reports have also linked glycine levels to visceral adipose tissue [24]. In a cross-sectional research enrolling 73 elderly people, glycine was negatively associated with visceral adipose tissue and HOMA-IR, and positively associated with subcutaneous adipose tissue [20]. Glycine was suggested as a marker of insulin sensitivity and was shown to reduce fat mass and insulin levels in rodents [31, 32]. The mechanisms involved in these positive effects of glycine are improved insulin sensitivity [33], and increased anti-inflammatory and antioxidant capacity [34, 35]. These results support our findings on a negative association between VFA and glycine levels. The mechanisms involved in plasma AA changes associated with abdominal obesity have not yet been clarified. Dietary intake, tissue catabolism, and protein synthesis contribute to AA concentrations [10]. Branched-chain AAs, which have been extensively studied in association with obesity and body fat distribution, are catabolized in visceral and subcutaneous adipose tissue [36]. Rodent models showed a defective expression of branched-chain AAs catabolic enzymes in fat tissue and increased protein degradation and pointed toward these changes as partially responsible for increased AA levels [37-39]. Also, human studies showed a lower expression of branched-chained AAs catabolic enzymes in visceral adipose tissue of obese women with metabolic syndrome as compared to healthy obese women [36]. However, the mechanism involved in the alteration of branched-chained AAs catabolic gene expression is unknown. Visceral adipose tissue is associated with an increased level of oxidative stress and previous studies reported increased cysteine levels associated with oxidative stress [40], thus supporting our findings of increased cysteine and tyrosine levels independent of insulin resistance [41].

Our study has several limitations that we must acknowledge. First, this study was a cross-sectional one and, thus, no causal relationship between AA levels and visceral adipose tissue can be purported. Also, the small sample size may have limited the accuracy of the methods in identifying the AAs associated with visceral adipose tissue. Another limitation is the inclusion of women only, meaning that our findings cannot be generalized to men. Although we assessed the intake of food supplements (including those containing AAs), no data was collected regarding dietary protein intake and physical exercise. This is a limitation as dietary intake of proteins may have influenced the level of assessed AAs. In conclusion, in this research, we identified metabolic signatures of visceral fat tissue. Although previous research found various metabolites to be associated with both visceral adiposity and insulin resistance, to our knowledge, the current study is the first attempt to identify those AAs that are associated with visceral adiposity independent of insulin resistance. The 5 AAs identified in our investigation may represent suitable candidates for future research as biomarkers of increased visceral adiposity and a generalized metabolic change that is associated with it.

\section{Abbreviations}

AA amino acids

BMI body mass index

FDR false discovery rate

HOMA-IR homeostatic model assessment for insulin resistance 
KEGG Kyoto Encyclopedia of Genes and Genomes

PC principal component

PCA principal component analysis

PLS-DA partial least-squares discriminant analysis

UHPLC ultrahigh performance liquid chromatography

UHPLC-Q-TOF -[ESI+]-MS ultrahigh performance liquid chromatography (UHPLC) coupled with electrospray ionization-quadrupole-time of MS in positive mode

VFA visceral fat area

VIP variable importance in the projection

\section{Acknowledgments}

This research was funded by Iuliu Hatieganu University of Medicine and Pharmacy, Cluj-Napoca, Romania, internal grant no. 7690/82/15.04.2016 and 5200/71/1.03.2017.

\section{Author Contributions}

AAM, GR: Conceptualization; AAM, CS, CGB, NH, GR: Methodology; AAM, AR, RMP, CLV, ICB, CS, CGB: Formal analysis; AAM, AR, RMP, CLV, NH, ICB, CS, CGB: Investigation; AAM: Resources; AAM, AR: Writing - original draft preparation; RMP, CLV, NH, ICB, CS, CGB, GR: Writing - review \& editing.

\section{Conflicts of Interest}

CGB. reports fees from AstraZeneca, Bayer, Boehringer Ingelheim, Eli Lilly, Novo Nordisk, Medtronic, and Sanofi; CLV received fees from AstraZeneca and Mylan; NH reports fees from AstraZeneca, Eli Lilly, Novo Nordisk, Mylan, and Sanofi; GR reports fees from AstraZeneca, Boehringer Ingelheim, Eli Lilly, Medtronic, MSD, Novo Nordisk, Roche, and Sanofi; AAM, ICB, CS, RMP and AR have nothing to disclose.

\section{References}

1. World Health Organization. Obesity and overweight. Available online: https:/www.who.int/news-room/factsheets/detail/obesity-and-overweight. (accessed on 7 August 2021).

2. Ard JD. Obesity. In: Heimburger DC, Ard JD (Eds). Handbook of Clinical Nutrition, 4th ed. Mosby, 2006, pp. 371-400. DOI: 10.1016/B978-0-323-039529.50023-4

3. Fox CS, Massaro JM, Hoffmann U, Pou KM, Maurovich-Horvat $\mathrm{P}$, Liu CY, et al. Abdominal visceral and subcutaneous adipose tissue compartments: association with metabolic risk factors in the Framingham Heart Study. Circulation 2007 Jul;116(1):39-48. DOI: 10.1161/CIRCULATIONAHA.106.675355

4. Kuk JL, Katzmarzyk PT, Nichaman MZ, Church TS, Blair SN, Ross, R. Visceral fat is an independent predictor of all-cause mortality in men. Obesity (Silver Spring). 2006 Feb;14(2):336-41. DOI: 10.1038/ oby. 2006.43

5. Piché ME, Tchernof A, Després JP. Obesity Phenotypes, Diabetes, and Cardiovascular Diseases. Circ Res. 2020 May;126(11):1477-500. DOI: 10.1161/CIRCRESAHA.120.316101

6. Purnell JQ. Definitions, Classification, and Epidemiology of Obesity. In: Purnell J, Laferrere B (Eds.); Obesity. https://www.endotext.org/section/obesity/. (accessed August, 30 2021). DOI: 10.2310/IM.1051

7. Neeland IJ, Boone SC, Mook-Kanamori DO, Ayers C, Smit RAJ, Tzoulaki I, et al. Metabolomics Profiling of Visceral Adipose Tissue: Results From MESA and the NEO Study. J Am Heart Assoc. 2019 May;8(9):e010810. DOI: 10.1161/JAHA.118.010810

8. Libert DM, Nowacki AS, Natowicz MR. Metabolomic analysis of obesity, metabolic syndrome, and type 2 diabetes: amino acid and acylcarnitine levels change along a spectrum of metabolic wellness. PeerJ. 2018 Aug;6:e5410. DOI: 10.7717/peerj.5410

9. Cernea S, Both E, Fodor A. The association of anthropometric parameters with markers of insulin and leptin secretion and resistance in type 2 diabetes mellitus. Rev Romana Med Lab. 2020;28(3):299-314. DOI:10.2478/ rrlm-2020-0028

10. Piro MC, Tesauro M, Lena AM, Gentileschi P, Sica G, Rodia $\mathrm{G}$, et al. Free-amino acid metabolic profiling of visceral adipose tissue from obese subjects. Amino Acids. 2020 Aug;52(8):1125-37. DOI: 10.1007/s00726020-02877-6

11. Fiehn O, Garvey WT, Newman JW, Lok KH, Hoppel CL, Adams SH. Plasma metabolomic profiles reflective of glucose homeostasis in non-diabetic and type 2 diabetic obese African-American women. PLoS One. 2010 Dec;5(12):e15234. DOI: 10.1371/journal. pone. 0015234 
12. Würtz P, Soininen $P$, Kangas AJ, Rönnemaa T, Lehtimäki T, Kähönen $\mathrm{M}$, et al. Branched-chain and aromatic amino acids are predictors of insulin resistance in young adults. Diabetes Care. 2013 Mar;36(3):648-55. DOI: $10.2337 / \mathrm{dc} 12-0895$

13. Morris C, O'Grada C, Ryan M, Roche HM, Gibney MJ, Gibney ER, et al. The relationship between BMI and metabolomic profiles: a focus on amino acids. Proc Nutr Soc. 2012 Nov;71(4):634-8. DOI: 10.1017/ S0029665112000699

14. She P, Van Horn C, Reid T, Hutson SM, Cooney RN, Lynch CJ. Obesity-related elevations in plasma leucine are associated with alterations in enzymes involved in branched-chain amino acid metabolism. Am. J. Physiol. Am J Physiol Endocrinol Metab. 2007 Dec;293(6):E1552-63. DOI: 10.1152/ajpendo.00134.2007

15. Geidenstam N, Magnusson M, Danielsson APH, Gerszten RE, Wang TJ, Reinius LE, et al. Amino Acid Signatures to Evaluate the Beneficial Effects of Weight Loss. Int J Endocrinol. 2017;2017:6490473. DOI: $10.1155 / 2017 / 6490473$

16. Wishart DS, Jewison T, Guo AC, Wilson M, Knox C, Liu Y, et al. HMDB 3.0--The Human Metabolome Database in 2013. Nucleic Acids Res. 2013 Jan;41:D801-7. DOI: $10.1093 /$ nar/gks 1065

17. Matthews DR, Hosker JP, Rudenski AS, Naylor BA, Treacher DF, Turner RC. Homeostasis model assessment: insulin resistance and beta-cell function from fasting plasma glucose and insulin concentrations in man. Diabetologia. 1985 Jul;28(7):412-9. DOI: 10.1007/BF00280883

18. Bala CG, Rusu A, Ciobanu D, Bucsa C, Roman G. Amino Acid Signature of Oxidative Stress in Patients with Type 2 Diabetes: Targeted Exploratory Metabolomic Research. Antioxidants (Basel). 2021 Apr;10(4):610. DOI: 10.3390/antiox 10040610

19. Liu X, Gao J, Chen J, Wang Z, Shi Q, Man H, et al. Identification of metabolic biomarkers in patients with type 2 diabetic coronary heart diseases based on metabolomic approach. Sci Rep. 2016 Jul;6:30785. DOI: 10.1038/srep30785

20. Lustgarten MS, Price LL, Phillips EM, Fielding RA. Serum glycine is associated with regional body fat and insulin resistance in functionally-limited older adults. PLoS One. 2013 Dec;8(12):e84034. DOI: 10.1371/ journal.pone.0084034

21. Boulet MM, Chevrier G, Grenier-Larouche T, Pelletier M, Nadeau M, Scarpa J, et al. Alterations of plasma metabolite profiles related to adipose tissue distribution and cardiometabolic risk. Am J Physiol Endocrinol Metab. 2015 Oct;309(8):E736-46. DOI: 10.1152/ajpendo.00231.2015

22. Martin FP, Montoliu I, Collino S, Scherer M, Guy P, Tavazzi I, et al. Topographical body fat distribution links to amino acid and lipid metabolism in healthy obese women. PLoS One. 2013 Sep;8(9):e73445. DOI: 10.1371/journal.pone.0073445

23. Yamakado M, Tanaka T, Nagao K, Ishizaka Y, Mitushima T, Tani M, et al. Plasma amino acid profile is associated with visceral fat accumulation in obese Japanese subjects. Clin Obes. 2012 Feb;2(1-2):29-40. DOI: 10.1111/j.1758-8111.2012.00039.x

24. Papandreou C, García-Gavilán J, Camacho-Barcia L, Hansen TT, Sjödin A, Harrold JA, et al. Circulating Metabolites Associated with Body Fat and Lean Mass in Adults with Overweight/Obesity. Metabolites. 2021 May;11(5):317. DOI: 10.3390/metabo11050317

25. Flores-Guerrero JL, Osté MCJ, Kieneker LM, Gruppen EG, Wolak-Dinsmore J, Otvos JD, et al. Plasma Branched-Chain Amino Acids and Risk of Incident Type 2 Diabetes: Results from the PREVEND Prospective Cohort Study. J Clin Med. 2018 Dec;7(12):513. DOI: $10.3390 /$ jcm 7120513

26. Tobias DK, Lawler PR, Harada PH, Demler OV, Ridker PM, Manson JE, et al. Circulating Branched-Chain Amino Acids and Incident Cardiovascular Disease in a Prospective Cohort of US Women. Circ Genom Precis Med. 2018 Apr;11(4):e002157. DOI: 10.1161/CIRCGEN.118.002157

27. Yang P, Hu W, Fu Z, Sun L, Zhou Y, Gong Y, et al. The positive association of branched-chain amino acids and metabolic dyslipidemia in Chinese Han population. Lipids Health Dis. 2016 Jul;15:120. DOI: 10.1186/ s12944-016-0291-7

28. Shah SH, Kraus WE, Newgard CB. Metabolomic profiling for the identification of novel biomarkers and mechanisms related to common cardiovascular diseases: form and function. Circulation. 2012 Aug;126(9):1110-20. DOI: 10.1161/CIRCULATIONAHA.111.060368

29. Hellmuth C, Kirchberg FF, Lass N, Harder U, Peissner W, Koletzko B, et al. Tyrosine Is Associated with Insulin Resistance in Longitudinal Metabolomic Profiling of Obese Children. J Diabetes Res. 2016;2016:2108909. DOI: $10.1155 / 2016 / 2108909$

30. Wang TJ, Larson MG, Vasan RS, Cheng S, Rhee EP, McCabe E, et al. Metabolite profiles and the risk of developing diabetes. Nat Med. 2011 Apr;17(4):448-53. DOI: $10.1038 / \mathrm{nm} .2307$

31. El Hafidi M, Pérez I, Zamora J, Soto V, Carvajal-Sandoval $\mathrm{G}, \mathrm{Ba}-\mathrm{os} \mathrm{G}$. Glycine intake decreases plasma free fatty acids, adipose cell size, and blood pressure in sucrose-fed rats. Am J Physiol Regul Integr Comp Physiol. 2004 Dec;287(6):R1387-93. DOI: 10.1152/ ajpregu.00159.2004

32. Alvarado-Vásquez N, Zamudio P, Cerón E, Vanda B, Zenteno E, Carvajal-Sandoval G. Effect of glycine in streptozotocin-induced diabetic rats. Comp. Biochem. Physiol. C. Comp Biochem Physiol C Toxicol Phar- 
macol. 2003 Apr;134(4):521-7. DOI: 10.1016/S15320456(03)00046-2

33. Gannon MC, Nuttall JA, Nuttall FQ. The metabolic response to ingested glycine. Am J Clin Nutr. 2002 Dec;76(6):1302-7. DOI: $10.1093 / \mathrm{ajcn} / 76.6 .1302$

34. Cruz M, Maldonado-Bernal C, Mondragón-Gonzalez R, Sanchez-Barrera R, Wacher NH, Carvajal-Sandoval $\mathrm{G}$, et al. Glycine treatment decreases proinflammatory cytokines and increases interferon-gamma in patients with type 2 diabetes. J Endocrinol Invest. 2008 Aug;31(8):694-9. DOI: 10.1007/BF03346417

35. Sekhar RV, McKay SV, Patel SG, Guthikonda AP, Reddy VT, Balasubramanyam A, et al. Glutathione synthesis is diminished in patients with uncontrolled diabetes and restored by dietary supplementation with cysteine and glycine. Diabetes Care. 2011 Jan;34(1):162-7. DOI: $10.2337 / \mathrm{dc} 10-1006$

36. Lackey DE, Lynch CJ, Olson KC, Mostaedi R, Ali M, Smith WH, et al. Regulation of adipose branched-chain amino acid catabolism enzyme expression and cross-adipose amino acid flux in human obesity. Am J Physiol Endocrinol Metab. 2013 Jun;304(11):E1175-87. DOI: 10.1152/ajpendo.00630.2012

37. She P, Van Horn C, Reid T, Hutson SM, Cooney RN, Lynch CJ. Obesity-related elevations in plasma leucine are associated with alterations in enzymes involved in branched-chain amino acid metabolism. Am J Physiol Endocrinol Metab. 2007 Dec;293(6):E1552-63. DOI: 10.1152/ajpendo.00134.2007

38. Herman MA, She P, Peroni OD, Lynch CJ, Kahn BB. Adipose tissue branched chain amino acid (BCAA) metabolism modulates circulating BCAA levels. J Biol Chem. 2010 Apr;285(15):11348-56. DOI: 10.1074/jbc. M109.075184

39. Pietiläinen KH, Naukkarinen J, Rissanen A, Saharinen $\mathrm{J}$, Ellonen $\mathrm{P}$, Keränen $\mathrm{H}$, et al. Global transcript profiles of fat in monozygotic twins discordant for BMI: pathways behind acquired obesity. PLoS Med. 2008 Mar;5(3):e51. DOI: 10.1371/journal.pmed.0050051

40. Samiec PS, Drews-Botsch C, Flagg EW, Kurtz JC, Sternberg P Jr, Reed RL, et al. Glutathione in human plasma: decline in association with aging, age-related macular degeneration, and diabetes. Free Radic Biol Med. 1998 Mar;24(5):699-704. DOI: 10.1016/S08915849(97)00286-4

41. Candi E, Tesauro M, Cardillo C, Lena AM, Schinzari F, Rodia G, et al. Metabolic profiling of visceral adipose tissue from obese subjects with or without metabolic syndrome. Biochem J. 2018 Mar;475(5):1019-35. DOI: 10.1042/BCJ20170604 